\title{
Erratum to: Hemispheric activation differences in novice and expert clinicians during clinical decision making
}

\author{
Pam Hruska $^{1}$ - Kent G. Hecker ${ }^{1,2}$ - Sylvain Coderre ${ }^{3}$ • \\ Kevin McLaughlin ${ }^{3} \cdot$ Filomeno Cortese $^{4} \cdot$ Christopher Doig $^{5}$ • \\ Tanya Beran $^{1} \cdot$ Bruce Wright $^{6} \cdot$ Olav Krigolson $^{7}$
}

\section{Erratum to: Adv in Health Sci Educ (2016) 21:921-933 DOI 10.1007/s10459-015-9648-3}

In order to more accurately reflect the 3D coordinates listed within this article, the column headings should be changed from "MNI coordinates" to "vox coordinates" in Table 1 (p. 927) and Table 2 (p. 928). If you would like the MNI coordinates for the Tables, please contact Dr. Kent Hecker.

The online version of the original article can be found under doi:10.1007/s10459-015-9648-3.

Kent G. Hecker

kghecker@ucalgary.ca

1 Department of Community Health Sciences, Cumming School of Medicine, University of Calgary, Calgary, AB, Canada

2 Department of Veterinary Clinical and Diagnostic Sciences, Faculty of Veterinary Medicine, University of Calgary, Calgary, AB, Canada

3 Undergraduate Medical Education, Cumming School of Medicine, Calgary, AB, Canada

4 Seaman Family MR Research Centre, Hotchkiss Brain Institute, University of Calgary, Calgary, AB, Canada

5 Department of Critical Care Medicine, Cumming School of Medicine, Calgary, AB, Canada

6 Division of Medical Sciences, University of Victoria, Victoria, BC, Canada

7 Neuroscience Program, Centre for Biomedical Research, School of Exercise Science, Physical, and Health Education, University of Victoria, Victoria, BC, Canada 